\title{
In vitro Root Architectural Screening for Early Drought Adaptive Traits in Sorghum (Sorghum bicolor L.)
}

\author{
Williams Mohanavel $^{1}$, N. Manikanda Boopathi ${ }^{1 *}$, \\ Raveendran Muthurajan ${ }^{1}$ and A. Senthil ${ }^{2}$
}

${ }^{1}$ Department of Plant Biotechnology, Center for Plant Molecular Biology and Biotechnology,

${ }^{2}$ Department of Crop Physiology, Tamil Nadu Agricultural University, Coimbatore 641003, India

*Corresponding author

\section{A B S T R A C T}

\begin{tabular}{l} 
K e y w o r d s \\
Root in-vitro study, \\
Root architecture, \\
Large scale \\
phenotypic \\
screening, \\
Sorghum, \\
\hline Article Info \\
\hline $\begin{array}{l}\text { Accepted: } \\
\text { 26 April } 2020 \\
\text { Available Online: } \\
\text { 10 May } 2020\end{array}$ \\
\hline
\end{tabular}

Drought is a major abiotic stress that causes a great reduction in the yield of the key cereal crops including sorghum. As the crops are sessile, they evolved several drought tolerance component traits to combat the water stress. Among the drought adaptive traits, root architecture plays a major role in efficient capillary water absorption even under severe water scarce situations through early development and deep penetration of roots. Thus, introgression of root traits in the elite breeding lines would enhance the breeding efficiency for water-limited environments. On the other hand, when compared with other drought tolerance component traits, phenotyping of root architecture is extremely challenging as it involves extensive excavation and destructive methodologies. Hence, a simple and efficient in vitro root architectural phenotyping in sorghum has been proposed in this study. Surface sterilization of sorghum seeds with $0.1 \% \mathrm{HgCl}_{2}$ and subsequent inoculation of the seeds in $1 / 2$ MS media provided effective visualization of root development and further analysis of root architecture traits. Such effort has helped to assess the significant genetic variation among ten different germplasm accessions used in this investigation. We suggest that such an in vitro screening methodology which is utilized to capture initial root development can be used as a rapid, nondestructive and efficient high-throughput tool to select the early drought adaptive lines among the large numbers of plant breeding materials.

\section{Introduction}

Drought is the most devastating stress which causes a severe decline in crop yield than any other environmental stress. The ability of plants to access water from deep soil solely depends on the root system development. In water-limited conditions, the capacity of the root system plays a critical role to adapt the situation. Maize and sorghum are the best examples that can switch to the water stress condition.

Sorghum produces solitary seminal root to absorb water and nutrient during the initial stage and to sustain in the later stage, it produces nodal root at $4^{\text {th }}$ to $5^{\text {th }}$ leaf stage (Singh et al., 2010). 
For increasing the accessibility of water by the plants under water stress condition, root architecture properties (such as size, length and distribution) plays an imperative role (Smith et al., 2005). To design a successful breeding strategy that incorporates desirable root traits, effective rapid phenotypic screening is essential which should focus on the nature of the root development at young stage and architecture of root would be essential. Several studies have been reported elsewhere on the plant response to the drought stress at the root level.

According to Tron et al., (2015), the influence of a root trait on drought tolerance will differ conditioning on the pattern and degree of water available in the soil. Lynch and van Beem (1993) reported that deep root system was mainly developed in plants under terminal drought condition and Wasson et al., (2012) observed that shallow root system was developed in plants where water was available in the topsoil.

Further, it was observed in sorghum, wheat and barley that the ability of the crop to absorb water was increased significantly with an increase in root length density (root length per unit volume) and specific root length Carvalho et al., (2014).

Similarly, the root angle also plays a significant role in drought tolerance. Manschadi et al., (2006) observed in the seedling stagethatthe angle at which root axes appeared was subsequently associated with root architecture and water uptake.

Hammer et al., (2009) reported similar pattern of water extraction from soil differs based on the root angle. Singh et al., (2010) further suggested that root angle and nodal root diameter could be used as a potential selection index for large scale screening of breeding population for tolerance under water-limited conditions. However, the roots system may get damaged while taken out of the soil for root architecture studies. Alternatively, screening of the root architecture can be done easily and effectively when they are grown in gel chamber ((Bengough et al., 2004; Zhu et al., 2019) and this method offers several advantages: root growth and morphology can be monitored easily without any interference and it provides a solid clarity in rhizosphere which allows measurement of complex traits in three dimensions (Judd et al., 2015).

On the other hand, concurrent results cannot be obtained between roots developed under soil and gel systems since the root development in soil is affected by several factors such as soil structure, moisture level, hardpan etc. (Gregory, 2009).

In soil-based study, root system investigation will interfere with soil particles and the reliability of the results depends on the roots without any physical damage. The complexity of the soil environment along with the geometric and physiological complexity of architectural traits are the other limitation of the root researches in soil-based root study (Lynch, 1995).

Thus, the use of the gel chamber system is recognized as a rapid and efficient methodology for high-throughput screening of breeding materials for the initial development of root traits. Further, with the advancement in information technology, roots in the gel chamber can be rapidly and precisely evaluated for root architecture using "imageJ" software (Abramoff MD, 2004). Hence, this study was designed with the aim of developing rapid in vitro screening methodology for sorghum initial root architecture which will be used to narrow down the initial large numbers of breeding materials into a workable size for drought tolerance improvement. 


\section{Materials and Methods}

\section{Plant materials}

We used uniform-sized seeds of ten sorghum germplasm lines (viz., IS 10803, IS 10815, IS 10889, IS 12150, IS 14276, IS 11818, IS 15146, IS 9970, IS 9912 and IS 6997) that were obtained from Indian Institute of Millets Research (IIMR), Hyderabad, India for this in vitro root investigation.

\section{Surface sterilization}

Initially surface sterilization of sorghum seeds was carried out under a laminar airflow chamber with different chemicals to identify the appropriate sterilizing agent and concentration. Sorghum seeds were washed with sterile water for 2-3 times and they were subjected to disinfectant agent viz., $\mathrm{NaOCl}$, $\mathrm{HgCl}_{2}$ and $\mathrm{H}_{2} \mathrm{O}_{2}$ at different concentrations and exposure time as outlined in table 1 . In all the sterilization procedure, Tween 20 was added, as a surface tension reducing agent, to improve seed surface contact with the disinfectant (Mekonnen et al., 2013). After the incubation period, seeds were washed thrice with sterile water and then dried in sterile tissue paper.

\section{In vitro phenotyping of root traits}

Acid washed boiling tubes $(30 \mathrm{~cm}$ longand5 $\mathrm{cm}$ diameter) were sterilized in a hot air oven at $150^{\circ} \mathrm{C}$ for 8 hours and then autoclaved at $121^{\circ} \mathrm{C}$ with $15 \mathrm{psi}$ for 20 minutes. Half strength MS media was prepared along with $3 \%$ sucrose $(30 \mathrm{~g} / \mathrm{l})$ and $\mathrm{pH}$ was adjusted to 5.8 using $1 \mathrm{~N} \mathrm{HCl}$ and $1 \mathrm{~N} \mathrm{NaOH}$. Finally, $0.3 \%$ Gelrite (3 g/l) was added as a solidifying agent, which provided a transparent media that facilitates easy visualizing of the root architecture. The media was heated for thorough mixing and poured into a boiling tube up to $15 \mathrm{~cm}$ (which needs $200 \mathrm{ml}$ of media), plugged with sterilized cotton plug and then autoclaved. After autoclaving, the tubes were allowed to solidify in the aseptic culture room and kept for four days to test, if there was any visibility of contamination.

\section{Inoculation of seeds}

Each sterilized sorghum germplasm accession's seeds were inoculated in the above media using sterile forceps under the laminar airflow chamber and after that they were left undisturbed for germination under dark condition. Once, the seeds were germinated, the tubes were brought to 12 hours' light (under normal white tube light that provided $~ 1000$ lux) for further growth and development. Regular statistical analysis (such as mean, standard deviation and standard error) of the data collected from three replications was done using Microsoft Office 2010 Excel.

\section{Root architecture analysis}

Fourteen days after sowing (DAS), photographs of the roots of each accession were taken at the same fixed position using a tripod stand and the photographs were used to measure the root architectural traits. Photographs were analyzed under "imageJ" a java based software for the root traits(Abramoff, 2004).

ObjectJ plugin was also added to the imageJ software for adjusting the image analysing parameters. Scales were set to convert pixel to the desired unit (cm, in this case) using the line tool of imageJ. Thus maximum root length, number of nodal roots, number of lateral roots, root angle and root width were measured for all the investigated sorghum germplasm accessions in triplicate and simple statistical analysis of root traits were done using Microsoft Office 2010 Excel. 


\section{Results and Discussion}

Generally, contamination that occurs during in vitro studies are attributed to different sources such as explant/seed, media, air and handling error. This investigation has compared the effect of three disinfectants $\left(\mathrm{HgCl}_{2}, \mathrm{NaOCl}\right.$ and $\left.\mathrm{H}_{2} \mathrm{O}_{2}\right)$ with different concentrations and treatment timing on microbial contamination. Among these disinfectants, it was evident that sorghum seed treatment with $0.1 \% \mathrm{HgCl}_{2}$ for 8 minutes provided the best contamination-free germination $(93.3 \%$ survival of explant with only $3 \%$ of contamination). In general, sterilizing agents inactivate or block the organelle or biochemical pathway of microorganisms and thereby minimizes the contamination (Groach et al., 2016). The highest contamination $(70 \%)$ with the lowest survival rate $(16 \%)$ was recorded when the seeds were surface sterilized with $10 \% \mathrm{H}_{2} \mathrm{O}_{2}$ for 10 minutes which may be due to the overdose of concentration and exposure duration.Similarly,56\% and $33 \%$ of contaminations were observed with $33 \%$ and $27 \%$ of survival of sorghum seeds at $5 \%$ and $10 \% \mathrm{NaOCl}$ concentrations, respectively. Nevertheless, the death of the explants was high indicating lower survival of explants in $10 \% \mathrm{NaOCl}$.

Fourteen days after inoculation, root traits such as maximum root length, number of lateral roots, number of nodal roots, root angle and root system width have been recorded using image $\mathbf{J}$ software and they were subjected to statistical analysis (Figure 1; Table 2). Among the ten different accessions, IS 10815 was having maximum root length $(14.69 \mathrm{~cm})$, more number of lateral root (20 nos) and highest system width $(2.98 \mathrm{~cm})$. Root angle which is an important trait in root architecture was highest in the accession IS $10889\left(116.65^{\circ}\right)$. Accession IS 10803 was having the lowest number of lateral roots ( 2 nos) with smallest root length $(2.71 \mathrm{~cm})$. Though accession IS 10889 was having a higher root angle, root length and number of lateral roots were very low (Table 2).

\section{Discussion}

The development of rapid and efficient root architecture assays has immense potential in screening of a large number of breeding materials for drought tolerance improvement in sorghum. Especially, the sorghum lines that have shown significant initial root system development can survive early-season drought stress by extracting as much water as possible from the available soil moisture. This study intended to use a transparent gel chamber technique for screening of root traits of sorghum among germplasm accessions.

The use of an effective disinfectant with an optimal concentration and length of exposure is an essential consideration for any planting of aseptic crops. $\mathrm{HgCl}_{2}$ was considered as a strong and effective sterilizing agent for the sorghum accessions due to its bactericidal activity. It was effective because of the bleaching action of two chloride atoms and the ions combine with protein causing the death of organisms (Mahmoud and Al-Ani, 2016). Among all the treatments, relatively improved survival rate of $93.3 \%$ was observed with $0.1 \% \mathrm{HgCl}_{2}$ for 8 minutes. Results of this study were in accordance with Alam et al., (2016) who have shown that increasing the duration of exposure had a negative effect on plant viability. With $0.2 \%$ $\mathrm{HgCl}_{2}$ for 5 minutes, contamination-free explants were observed but at the same time, the survival rate was not satisfactory.

Similar kind of decrease in viability of the explants at higher concentrations of sterilizing agent though it was free of contamination was reported by Groach et al., (2016). 
Table.1 Standardization of surface sterilizing agents for aseptic germination of sorghum seeds

\begin{tabular}{|c|c|c|c|c|c|c|c|c|}
\hline \multirow{2}{*}{ Protocol } & \multicolumn{2}{|c|}{ Ethanol } & \multicolumn{2}{c|}{ NaOCl } & \multicolumn{2}{c|}{$\mathbf{H g C l}_{\mathbf{2}}$} & \multicolumn{2}{c|}{$\mathbf{H}_{\mathbf{2}} \mathbf{O}_{2}$} \\
\cline { 2 - 11 } & $\%$ v/v & Duration & $\%$ v/v & Duration & $\%$ v/v & Duration & $\%$ v/v & Duration \\
\hline$* \mathbf{P 1}$ & $70 \%$ & $1 \mathrm{~min}$ & $5 \%$ & $15 \mathrm{mins}$ & - & - & - & - \\
\hline$* \mathbf{P 2}$ & $90 \%$ & $1 \mathrm{~min}$ & $10 \%$ & $15 \mathrm{mins}$ & - & - & - & - \\
\hline$* \mathbf{P 3}$ & $70 \%$ & $1 \mathrm{~min}$ & - & - & $0.2 \%$ & $5 \mathrm{mins}$ & - & - \\
\hline$* \mathbf{P 4}$ & $70 \%$ & $2 \mathrm{mins}$ & - & - & $0.1 \%$ & $8 \mathrm{mins}$ & - & - \\
\hline$* \mathbf{P 5}$ & $70 \%$ & $5 \mathrm{mins}$ & - & - & - & - & $10 \%$ & 10 mins \\
\hline
\end{tabular}

*Sterilization treatments applied to sorghum seeds using three different disinfectants viz., $\mathrm{NaOCl}, \mathrm{HgCl}_{2}$ and $\mathrm{H}_{2} \mathrm{O}_{2}$ are described in this table as $* \mathrm{P} 1-5 \% \mathrm{NaOCl}$ for 15 mins, $* \mathrm{P} 2-10 \% \mathrm{NaOCl}$ for 15 mins, $* \mathrm{P} 3-0.2 \% \mathrm{HgCl} 2$ for 5 minutes, *P4 - $0.1 \% \mathrm{HgCl} 2$ for 8 minutes, ${ }^{*} \mathrm{P} 5-10 \% \mathrm{HgCl} 2$ for 10 minutes, $\mathrm{C}$-Contamination and $\mathrm{S}$ - Survival

Table.2 Results of in vitro analysis root traits in sorghum germplasm

\begin{tabular}{|c|c|c|c|c|c|}
\hline Germplasm & MxRL(cm) & No.of LR & No.of NR & Root angle( & Sys width(cm) \\
\hline IS 10803 & 2.71 & 2.00 & 2.00 & 73.58 & 0.70 \\
\hline IS 10815 & 14.69 & 20.00 & 3.00 & 91.56 & 2.98 \\
\hline IS 10889 & 7.95 & 8.00 & 3.50 & 116.65 & 1.07 \\
\hline IS 12150 & 7.17 & 5.50 & 5.00 & 107.87 & 2.60 \\
\hline IS 14276 & 12.21 & 15.00 & 3.50 & 11.40 & 1.29 \\
\hline IS 11818 & 13.84 & 15.50 & 3.00 & 76.59 & 1.30 \\
\hline IS 15146 & 3.54 & 1.00 & 0.50 & 68.40 & 0.59 \\
\hline IS 9970 & 7.14 & 6.50 & 5.00 & 95.69 & 1.91 \\
\hline IS 9912 & 6.53 & 8.00 & 3.00 & 95.37 & 1.59 \\
\hline IS 6997 & 5.84 & 5.50 & 4.00 & 87.60 & 1.24 \\
\hline Mean & 8.16 & 8.70 & 3.25 & 92.47 & 1.53 \\
\hline SE & 1.30 & 1.96 & 0.42 & 5.18 & 0.24 \\
\hline SD & 4.12 & 6.18 & 1.34 & 16.37 & 0.77 \\
\hline Kurtosis & -0.91 & -0.49 & 1.01 & -1.14 & 0.01 \\
\hline Skewness & 0.49 & 0.69 & -0.67 & -0.01 & 0.86 \\
\hline
\end{tabular}

MxRL - Maximum Root length (cm); No.of LR - No. of Lateral root;

No.of NR - No. of Nodal root; Sys width - System width (cm); SE - Standard Error; SD - Standard Deviation

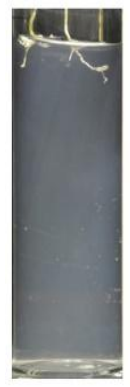

IS10803

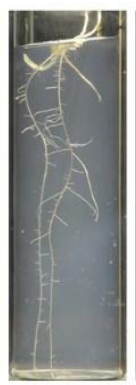

IS10815

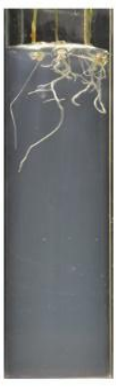

IS12150

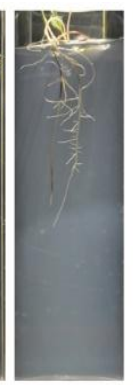

IS14276

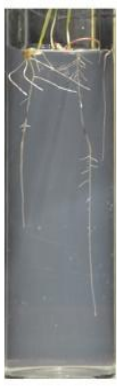

IS11818

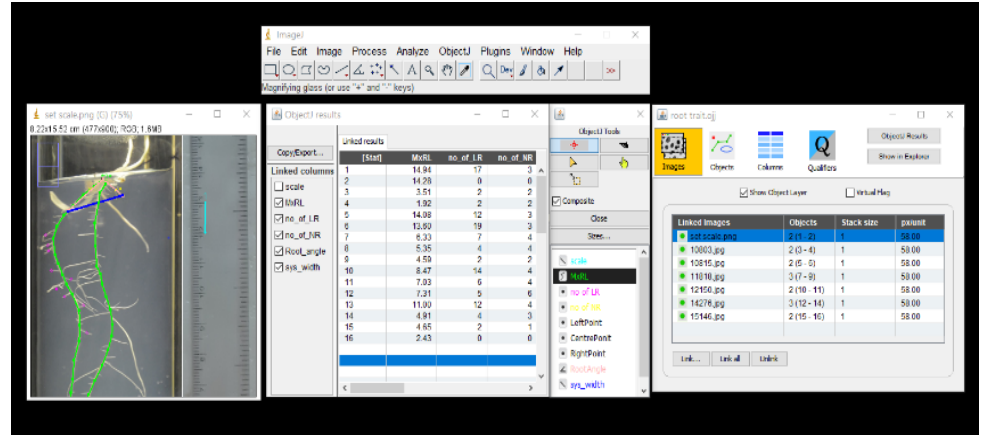

Figure.1 Results of analyzing the five different root traits using image $\mathrm{J}$ under object $\mathrm{J}$ plugin 
Hence, it was inferred that surface sterilization should eliminate contaminants to the maximum extent and it shouldn't have any impact on biological substance. $\mathrm{NaOCl}$ is a mild/ weak sterilizing agent so that it was needed in higher concentrations to maintain aseptic conditions. $\mathrm{NaOCl}$ with water form $\mathrm{HClO}$ that lead to a lethal DNA damage in a microorganism (Urbi and Zainuddin, 2015).

It was observed that when the concentration of $\mathrm{NaOCl}$ was increased from $5 \%$ to $10 \%$, the contamination rate was reduced by $23 \%$ however the survival rate was also reduced by $6 \%$. These results are in accordance with Hesami et al., (2017) who observed that the lowest contamination was recorded in a high concentration of $\mathrm{NaOCl}$ even up to $20-25 \%$ but seedling growth and viability get affected. Sen et al., (2013)further observed that seeds turn to black when they were treated with a high concentration of $\mathrm{NaOCl}$. Plant with maximum root length can absorb more water from the deep soild during drought stress conditions. Accession IS 10815 has maximum root length with increased number of lateral root which can efficiently absorb water from the soil. This accession also has the highest root system width which indicates the spreading pattern of roots. Similar kind of studies to identify a barley germplasm having highest root length in gel-based assay has been reported earlier (Hargreaves et al., 2009).

Thus, this research documented an effective protocol for surface sterilization of sorghum seeds for an in vitro root architecture analysis. It has been concluded that $0.1 \% \mathrm{HgCl}_{2}$ at 8 minutes was effective than other disinfecting agents such as $\mathrm{NaOCl}$ and $\mathrm{H}_{2} \mathrm{O}_{2}$. The gel based assay reported in this investigation has shown to be simple, easy and non-destructive method of screening root architectural traits. This approach can be used to screen the rootrelated traits of sorghum germplasm resources on a wide scale and can be used in crop improvement programs that focus on early drought tolerance.

\section{Acknowledgment}

The authors would like to thank Indian Institute of Millets Research for sharing the sorghum germplasm resources.

\section{References}

Abramoff MD, M.P., Ram SJ. 2004. "Image processing with ImageJ. Biophotonics International 11: 36-42."

Alam, F., E. Uddin, R. Amin, A. Razzak, M. Manik, and M. Khatu. 2016. "Studies on the effect of various sterilization procedure for in vitro seed germination and successful micropropagation of Cucumis sativus." Int. J. Pure App. Biosci 4 (1):75-81.

Bengough, A., D. Gordon, H. Al-Menaie, R. Ellis, D. Allan, R. Keith, W. Thomas, and B. Forster. 2004. "Gel observation chamber for rapid screening of root traits in cereal seedlings." Plant and Soil 262 (1-2):63-70.

Carvalho, P., S. Azam- Ali, and M.J. Foulkes. 2014. "Quantifying relationships between rooting traits and water uptake under drought in Mediterranean barley and durum wheat." Journal of integrative plant biology 56 (5):455-469.

Comas, L., S. Becker, and V. Cruz. "V., Byrne PF, Dierig DA. 2013. Root traits contributing to plant productivity under drought." Frontiers in plant science 4:1-16.

Gregory, P. 2009. "Measuring root system architecture: Opportunities and challenges." International Symposium "Root Research and Applications" Root RAP.

Hammer, G.L., Z. Dong, G. McLean, A. Doherty, C. Messina, J. Schussler, C. Zinselmeier, S. Paszkiewicz, and M. Cooper. 2009. "Can changes in canopy and/or root system architecture explain historical maize yield trends in the US corn belt?" Crop Science 49 (1): 299-312.

Hargreaves, C.E., P.J. Gregory, and A.G. Bengough. 2009. "Measuring root traits in barley (Hordeum vulgare ssp. vulgare and 
ssp. spontaneum) seedlings using gel chambers, soil sacs and X-ray microtomography." Plant and Soil 316 (12):285-297.

Hesami, M., M.H. Daneshvar, and A. LotfiJalalabadi. 2017. "Effect of sodium hypochlorite on control of in vitro contamination and seed germination of Ficus religiosa." Iranian Journal of Plant Physiology 7 (4).

Judd, L.A., B.E. Jackson, and W.C. Fonteno. 2015. "Advancements in root growth measurement technologies and observation capabilities for container-grown plants." Plants 4 (3):369-392.

Lynch, J. 1995. "Root architecture and plant productivity." Plant physiology 109 (1):7.

Lynch, J., and J.J. van Beem. 1993. "Growth and architecture of seedling roots of common bean genotypes." Crop Science 33 (6):1253-1257.

Mahmoud, S.N., and N.K. Al-Ani. 2016. "Effect of different sterilization methods on contamination and viability of nodal segments of Cestrum nocturnum L." International Journal of Research Studies in Biosciences (IJRSB) 4 (1):4-9.

Manschadi, A.M., J. Christopher, P. deVoil, and G.L. Hammer. 2006. "The role of root architectural traits in adaptation of wheat to water-limited environments." Functional plant biology 33 (9):823-837.

Mekonnen, T., M. Diro, and M. Sharma. 2013. "An alternative safer and cost effective surface sterilization method for sugarcane (Saccharum officinarum L.) explants." African Journal of Biotechnology 12 (44):6282-6286.
Sen, M.K., M. Jamal, and S. Nasrin. 2013. "Sterilization factors affect seed germination and proliferation of Achyranthes aspera cultured in vitro." Environmental and Experimental Biology 11:119-123.

Singh, V., E.J. van Oosterom, D.R. Jordan, C.D. Messina, M. Cooper, and G.L. Hammer. 2010. "Morphological and architectural development of root systems in sorghum and maize." Plant and Soil 333 (1-2):287299.

Smith, D., N. Inman-Bamber, and P. Thorburn. 2005. "Growth and function of the sugarcane root system." Field Crops Research 92 (2-3):169-183.

Tron, S., G. Bodner, F. Laio, L. Ridolfi, and D. Leitner. 2015. "Can diversity in root architecture explain plant water use efficiency? A modeling study." Ecological modelling 312:200-210.

Urbi, Z., and Z. Zainuddin. 2015. "Standardization of surface sterilization protocol of field grown Stevia rebaudiana prior to in vitro clonal propagation." Journal Teknologi 77 (24):141-146.

Wasson, A.P., R. Richards, R. Chatrath, S. Misra, S.S. Prasad, G. Rebetzke, J. Kirkegaard, J. Christopher, and M. Watt. 2012. "Traits and selection strategies to improve root systems and water uptake in water-limited wheat crops." Journal of experimental botany 63 (9):3485-3498.

Zhu, Y.H., J. Weiner, M.X. Yu, and F.M. Li. 2019. "Evolutionary agroecology: Trends in root architecture during wheat breeding." Evolutionary applications 12 (4):733-743.

\section{How to cite this article:}

Williams Mohanavel, N. Manikanda Boopathi, Raveendran Muthurajan and Senthil, A. 2020. In vitro Root Architectural Screening for Early Drought Adaptive Traits in Sorghum (Sorghum bicolor L.). Int.J.Curr.Microbiol.App.Sci. 9(05): 3161-3167.

doi: https://doi.org/10.20546/ijcmas.2020.905.375 